\title{
A Cerebellar Internal Models Control Architecture for Online Sensorimotor Adaptation of a Humanoid Robot Acting in a Dynamic Environment
}

\author{
Capolei, Marie Claire; Andersen, Nils Axel; Lund, Henrik Hautop ; Falotico, Egidio; Tolu, Silvia
}

Published in:

IEEE Robotics and Automation Letters

Link to article, DOI:

10.1109/LRA.2019.2943818

Publication date:

2020

Document Version

Peer reviewed version

Link back to DTU Orbit

Citation (APA):

Capolei, M. C., Andersen, N. A., Lund, H. H., Falotico, E., \& Tolu, S. (2020). A Cerebellar Internal Models Control Architecture for Online Sensorimotor Adaptation of a Humanoid Robot Acting in a Dynamic Environment. IEEE Robotics and Automation Letters, 5(1), 80-87. https://doi.org/10.1109/LRA.2019.2943818

\section{General rights}

Copyright and moral rights for the publications made accessible in the public portal are retained by the authors and/or other copyright owners and it is a condition of accessing publications that users recognise and abide by the legal requirements associated with these rights.

- Users may download and print one copy of any publication from the public portal for the purpose of private study or research.

- You may not further distribute the material or use it for any profit-making activity or commercial gain

- You may freely distribute the URL identifying the publication in the public portal 


\title{
A Cerebellar Internal Models Control Architecture for Online Sensorimotor Adaptation of a Humanoid Robot Acting in a Dynamic Environment
}

\author{
Marie Claire Capolei ${ }^{1}$, Nils Axel Andersen ${ }^{1}$, Henrik Hautop Lund ${ }^{1}$, Egidio Falotico ${ }^{2}$, and Silvia Tolu ${ }^{1}$
}

\begin{abstract}
Humanoid robots are often supposed to operate in non-deterministic human environments, and as a consequence, the robust and gentle rejection of the external perturbations is extremely crucial. In this scenario, stable and accurate behavior is mostly solved through adaptive control mechanisms that learn an internal model to predict the consequences of the outgoing control signals. Evidences show that brain-based biological systems resolve this control issue by updating an appropriate internal model that is then used to direct the muscles activities. Inspired by the biological cerebellar internal models theory, that couples forward and inverse internal models into the biological motor control scheme, we propose a novel methodology to artificially replicate these learning and adaptive principles into a robotic feedback controller. The proposed cerebellar-like network combines machine learning, artificial neural network, and computational neuroscience techniques to deal with all the nonlinearities and complexities that modern robotic systems could present. Although the architecture is tested on the simulated humanoid iCub, it can be applied to different robotic systems without excessive customization, thanks to its neural networkbased nature. During the experiments, the robot is requested to follow repeatedly a movement while it is interacting with two external systems. Four different internal model architectures are compared and tested under different conditions. The comparison of the performances confirmed the theories about internal models combinatory action. The combination of models together with the structural and learning features of the network, resulted in a benefit to the adaptation mechanism, but also the system response to nonlinearities, noise and external forces.
\end{abstract}

Index Terms-Biomimetics, Neurorobotics, Model Learning for Control, Learning and Adaptive Systems, Control Architectures and Programming.

\section{INTRODUCTION}

$\mathbf{M}$ ODERN robots are often mechanically complex, and are embedded in unstructured non-deterministic environments [1]. The accurate and stable motor control of such systems is often challenging due to the unreliability of the

Manuscript received: June, 24, 2019; Revised July, 26, 2019; Accepted September, 10, 2019.

This paper was recommended for publication by Editor Youngjin Choi upon evaluation of the Associate Editor and Reviewers' comments. This work has received funding from the EU-H2020 Framework Program for Research and Innovation under the specific grant agreement No. 785907 (Human Brain Project SGA2), and from the Marie Curie project n. 705100 (Biomodular).

${ }^{1}$ Marie Claire Capolei, Nils A. Andersen, Henrik Hautop Lund and Silvia Tolu are with the Automation and control group, Department of Electrical Engineering, Technical University of Denmark, Kgs. Lyngby, Denmark $\{$ macca, naa, hhl, stolu\}@elektro.dtu.dk

${ }^{2}$ Egidio Falotico is with the BioRobotics Institute, Scuola Superiore Sant'Anna, Pontedera, Pisa, Italy egidio.falotico@santannapisa.it

Digital Object Identifier (DOI): see top of this page. hand engineered modeling strategies, which are too strict to describe all the complexities and nonlinearities.

In this manuscript, we propose an online learning and control algorithm to dynamically adapt the movements of a robotic system acting in an uncertain non-deterministic environment. In the design process, we assumed that: the Jacobian poorly describes the actual robotic condition; one or more unmodeled external objects interfere with the movement; the state space system is multivariable and not fully observable; the action/state space is continuous and high-dimensional. In this view, the controller should improve the tracking accuracy of each actuator, and minimize the effects of noise through force-based control input.

Traditionally, uncertain systems were learned by estimating open parameters of structured mathematical models [2]. Although this approach has been used for several years in system identification and adaptive control, fitting the parameters of fixed structure with training data can lead to different drawbacks, such as: physical inconsistency [3]; unmodeled behavior; persistent excitation issues [4]; and unstable reaction to high estimation error.

In the last decades, due to the advancement in artificial intelligence, a large number of non-parametric approaches have been proposed to solve the aforementioned problems [5], [6], [7], [8], [9], [10]. For instance, the introduction of artificial neural networks (ANNs) into nonlinear dynamical systems adaptive control were advantageous for reducing the effects of nonlinearities and uncertainties, and for handling high dimensional and continuous state space systems [11], [12], [13], [8], [14]. Although the structural versatility that distinguishes ANNs, the continuous interaction within the robotic system and the non-deterministic environment can be constrained by the off-line training of the neural network.

The Autonomous Mental Development (AMD) theorists claim that robots should learn and evolve their processing through real-time interaction with the environment [15], [16]. In this view, model learning is not seen anymore as a summation of off-line learned experiences but as an online development of the current knowledge of the system [17], [18]. These theories have their foundation in studies of biological systems, such as humans, especially infants. The advanced mechanisms exploited by biological systems to explore their relation with the surroundings, and control their own movements, motivated several scientists towards a better understanding of the biological motor control.

James S. Albus was the first person to propose a robotic 
control architecture enhanced by an artificial neural network based on evidence of the central nervous system (CNS): the "cerebellar model articulation controller" (CMAC) [19]. The CMAC module was mainly inspired by the David Maar's theory [20] that depicts the cerebellum, a neural structure located at the back of the brain, as "language translator between data in the cerebrum, and command sequences needed by the muscles" [21].

In the last decades, several scientists have been attracted by the fascinating mechanisms and functional roles of the cerebellum in motor and cognitive tasks [22], [23], [24], [25], [26], [27]. Among all the hypotheses, the scientific community is highly supporting the involvement of the cerebellum in the acquisition and maintenance of the internal models, mapping the correlation within the body and the environment [28], [29], [30], [31], i.e., forward and inverse models [32], [33]. If confirmed, these assumptions would explain several complex mechanisms underlying the neural control of movements [34].

The inverse model elaborates the motor command that leads the system from the current state to a desired one [35]. Its contribution enables fast and coordinated limb movements, that are not achievable with pure feedback control, due to the biological system dynamics [32]. Evidences show that some of the motor deficits caused by cerebellar dysfunction, e.g., quick ballistic limbs movements and impaired muscle coordination [36], are due to the lack of feed forward contribution in motor control, or rather the neural control loop is affected by slow reaction time and sensory delay [34]. Although it is controversial [37], [38], scientists argued that integrating the efference copy signal of the delayed sensory feedback could overcome these CNS transmission problems [39]. Different prototypes of cerebellar control architecture based on the inverse model theory has been proposed, such as: adaptive filter models [40], [41]; spiking neural networks [42], [43]; combination of parametric adaptive control and machine learning techniques [44], [45].

The forward model describes the causal relationship between the outgoing motor command and system state. This model results beneficial to predict those state transitions that are not directly accessible [46]. Electrophysiological studies [47], [48], computational theories [28], [29], imaging and lesion data [49], [50] suggest that the forward model could explain pivotal cerebellar functions, such as error correction and learning. Moreover, robotics experiments proved that the forward model could play an important role in action prediction, sensory discrepancy minimization, and noise cancellation [51], [52].

Inspired by the theory of coupled internal models [53], [54], [55], [56], [57], [58], we propose a novel methodology to replicate and exploit artificially the cerebellar internal models learning and corrective action. In particular, we designed a neural network that, through the combination of machine learning, artificial neural network, and computational neuroscience techniques, replicates the functionality, learning, modularity, and morphology of the cerebellar-circuit. This biomimetic network is embedded in a feedback robotic control architecture, and is intended to minimize modeling errors and to constrain the effects of noise, uncertainties, and external dis-

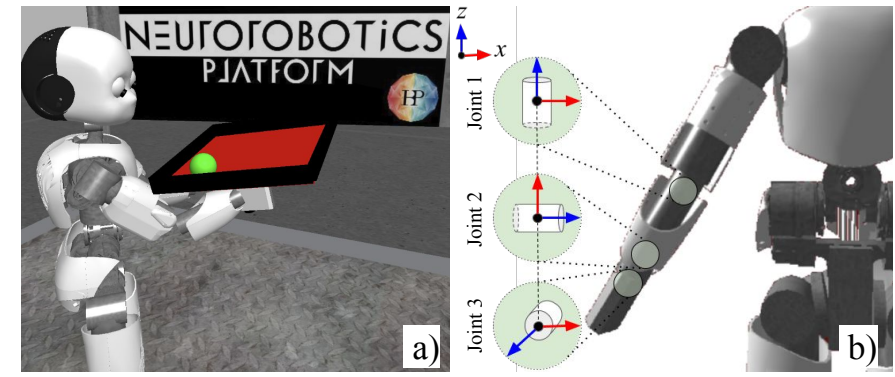

Fig. 1: Robotic plant: a) the humanoid iCub holding the table-ball system in the Neurorobotics Platform; b) the three controlled wrist joints: 1 pronosupination, 2 yaw, $\mathbf{3}$ pitch.

turbances. The network weights are defined by non-linear and multidimensional learning functions that mimic the cerebellar synaptic plasticities, as proposed by [59], [42]. The manuscript presents the comparison of four adaptive control architectures based on the cerebellar internal models theories. The control system is tested on the virtual humanoid robot iCub [60] embedded in the Neurorobotics Platform (Fig.1.a)[61], [62]. The architectures performance are evaluated under different noise and external perturbation conditions. The study confirmed that the forward and inverse internal model coupling shows improved performance respect to the independent internal models action. Moreover, the biologically plausible weighting kernel together with the layered structure of the cerebellar networks resulted beneficial to constrains the effects of external perturbations and nonlinearities.

The structure of the paper is as follows: in section II we describe the overall control architecture, giving special focus to the cerebellar-like component; in section III, the experimental set up and results are presented. The manuscript concludes with the discussion of the main findings in comparison with the literature and future directions.

\section{MATERIALS AND METHODS}

The robotic system, or rather Agent (Fig.2.a), consists of: a Planner, which generates the $\mathbf{Q}_{N \times 1}^{r}, \dot{\mathbf{Q}}_{N \times 1}^{r}$ reference motors angular positions and velocities (where $\mathrm{N}$ is the number of controlled joints), that are sent to the controller; the Controller, which elaborates the $\boldsymbol{\tau}_{N \times 1}^{t o t}$ torque commands needed to move the actuators to the $\mathbf{Q}_{N \times 1}^{r}, \dot{\mathbf{Q}}_{N \times 1}^{r}$ desired states; the Robotic Plant, which includes the actuators and the proprioceptive sensors employed to read the $\mathbf{Q}$ and $\dot{\mathbf{Q}}$ actual angular positions and velocities respectively. The Agent interacts with two external systems, which in this manuscript are represented by a table and a rolling ball (Fig.1.a).

\section{A. Robot Plant}

The humanoid iCub is a 53 degree of freedom (dof) robotic system equipped with several type of sensors, such as: encoders, accelerometers, gyroscopes, F/T sensors, digital cameras. For the sake of simplicity, the overall system actuates seven motors of the right arm: four motors are kept constant to keep the arm upwards (i.e. elbow, shoulder roll, shoulder yaw and shoulder pitch), and $N=3$ motors are controlled by 
the proposed controller (namely wrist pronosupination, wrist yaw and wrist pitch, Fig.1.b). The $n$-th actual motor state is read by the encoders and saved in the $q_{n} \in \mathbf{Q}_{N \times 1}$ angular position and $\dot{q}_{n} \in \dot{\mathbf{Q}}_{N \times 1}$ angular velocity process variables.

\section{B. Planner}

The Planner plans the $q_{n}{ }^{r} \in \mathbf{Q}_{N \times 1}^{r}, \dot{q}_{n}^{r} \in \dot{\mathbf{Q}}_{N \times 1}^{r}$ reference trajectories, or rather it generates oscillator movements,

$$
\begin{gathered}
q_{n}^{r}=A_{n} \cdot \sin \left(2 \pi f t+\varphi_{n}\right), \\
\dot{q}_{n}^{r}=2 \pi f A_{n} \cdot \cos \left(2 \pi f t+\varphi_{n}\right),
\end{gathered}
$$

with fixed temporal frequency $f=0.25 \mathrm{~Hz}, A_{n}$ amplitude and $\varphi_{n}$ phase,

$$
\begin{gathered}
\mathbf{A}_{1 \times N}=\left[A_{1}, A_{2}, A_{3}\right]=[0.1727,0.1363,0.0345] \mathrm{rad} \\
\varphi_{1 \times N}=\left[\boldsymbol{\varphi}_{1}, \varphi_{2}, \varphi_{3}\right]=\left[\begin{array}{ll}
0.5 \pi, 0.5 \pi, 0.0 & \mathrm{rad} .
\end{array}\right.
\end{gathered}
$$

\section{Controller}

The Controller once received the $\mathbf{Q}, \dot{\mathbf{Q}}$ actual robot states computes the $\tau_{n}{ }^{t o t} \in \boldsymbol{\tau}_{N \times 1}^{t o t}$ torque command to move each actuator to the $q_{n}^{r}, \dot{q}_{n}^{r}$ desired state. This subsystem is constituted by a static module based on classical control methods, and by two decentralized cerebellar-like neural networks (section II-D): inverse and forward models (blue boxes Fig.2.b). The inverse cerebellar-like module adds $\Delta \tau_{n}^{c} \in \Delta \boldsymbol{\tau}_{N \times 1}^{c}$ feed-forward corrective torque command to the $\tau_{n}^{f b}, \in \boldsymbol{\tau}_{N \times 1}^{f b}$ feedback controller motor input [63], [64], while the forward module applies $\Delta \dot{q}_{n}^{c} \in \boldsymbol{\Delta} \dot{\boldsymbol{q}}^{c}$ state-specific adjustment to the feedback loop [65], [66], [58]. In this initial design, the
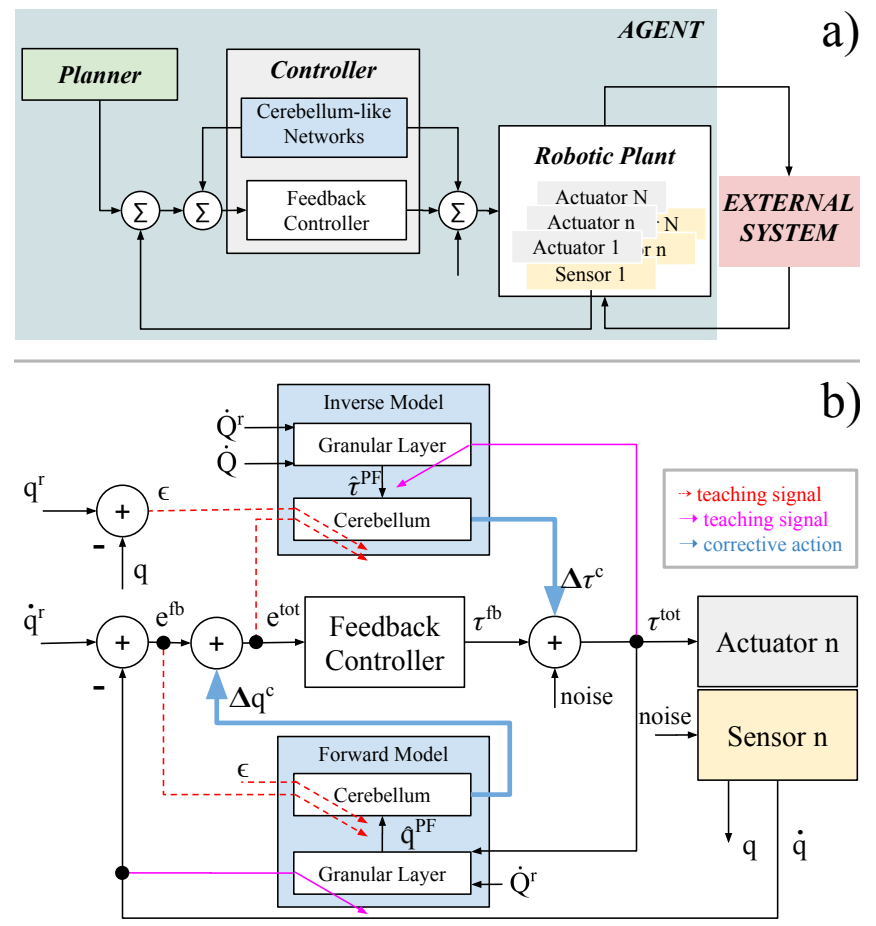

Fig. 2: Control architecture scheme for $\mathrm{N}$ actuated joints: a) main components communication, and b) controller block. forward model corrective term is narrowed to the angular velocity, which is the feedback controller input.

In the details of Fig.2.b, the closed-loop computes the $e_{n}^{f b} \in$ $\mathbf{e}_{N \times 1}^{f b}$ feedback angular velocity error of the $n$-th motor,

$$
e_{n}^{f b}=\dot{q}_{n}^{r}-\dot{q}_{n} .
$$

This quantity is corrected by the forward cerebellar-like module which predicts the consequence of the outgoing motor command and adds $\Delta \dot{q}_{n}^{c}$ contribution to minimize the $e_{n}^{f b}$ feedback error. The $\mathbf{e}^{t o t}$ total error,

$$
e_{n}^{t o t}=e_{n}^{f b}+\Delta \dot{q}_{n}^{c},
$$

it is then employed by both the feedback controller to compute the feedback torque command $\tau_{n}^{f b}$, according to the proportional-integrative-derivative (PID) independent joint control law, and by the inverse cerebellar-like model to compute the corrective torque $\Delta \tau_{n}^{c} \in \Delta \tau_{N \times 1}^{c}$, that minimizes both the $\mathbf{e}^{t o t}$ and the $\epsilon_{n} \in \boldsymbol{\epsilon}$ angular position error,

$$
\epsilon_{n}=q_{n}^{r}-q_{n} .
$$

The total control input sent to the motors is the result of a feed-forward compensation [40],

$$
\tau^{t o t}=\boldsymbol{\tau}^{f b}+\Delta \tau^{c} .
$$

On a final note, the PID regulator $\mathbf{K}$ gains are tuned to weakly operate in linearized conditions which exclude the disturbance of the ball and sensory noise,

$$
\begin{aligned}
& \mathbf{K}^{P}=\left[K_{1}^{P}, K_{2}^{P}, K_{3}^{P}\right]=[2.9000,2.3000,2.3500] \\
& \mathbf{K}^{I}=\left[K_{1}^{I}, K_{2}^{I}, K_{3}^{I}\right]=[1.9400,1.9000,1.9000] \\
& \mathbf{K}^{D}=\left[K_{1}^{D}, K_{2}^{D}, K_{3}^{D}\right]=[0.0050,0.0001,0.0004] \text {. }
\end{aligned}
$$

\section{Cerebellar-like Network}

The cerebellum is constituted of several micro-zones that plausibly correspond to the minimal ulm unit learning machine (Fig.3) [63]. Each ulm presents similar internal microcircuitry, but it differs from the others in terms of external connectivity. There are two main type of axons that connect each $u l m$ to the outside: the mf mossy fibers (in magenta Fig.3), which project signals regarding the position, velocity and direction of the limbs movements [68]; the climbing fibers (in red), that project from the io inferior olive nucleus the signal encoding the error [47], [69]. These axons transmits the information to two main groups of cells: the Gr granule

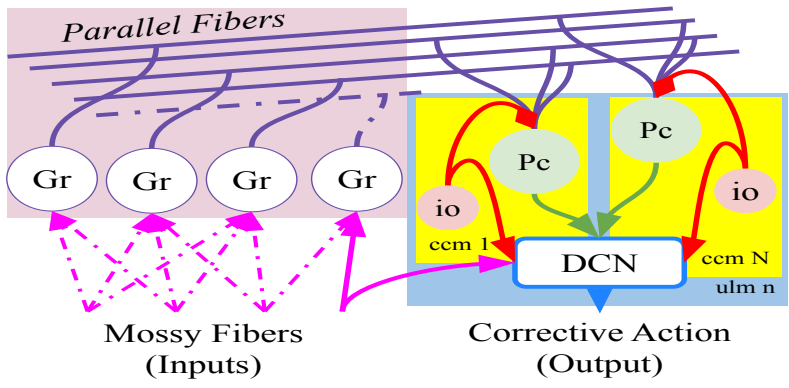

Fig. 3: Canonical cerebellar circuit in analogy with [67]. 


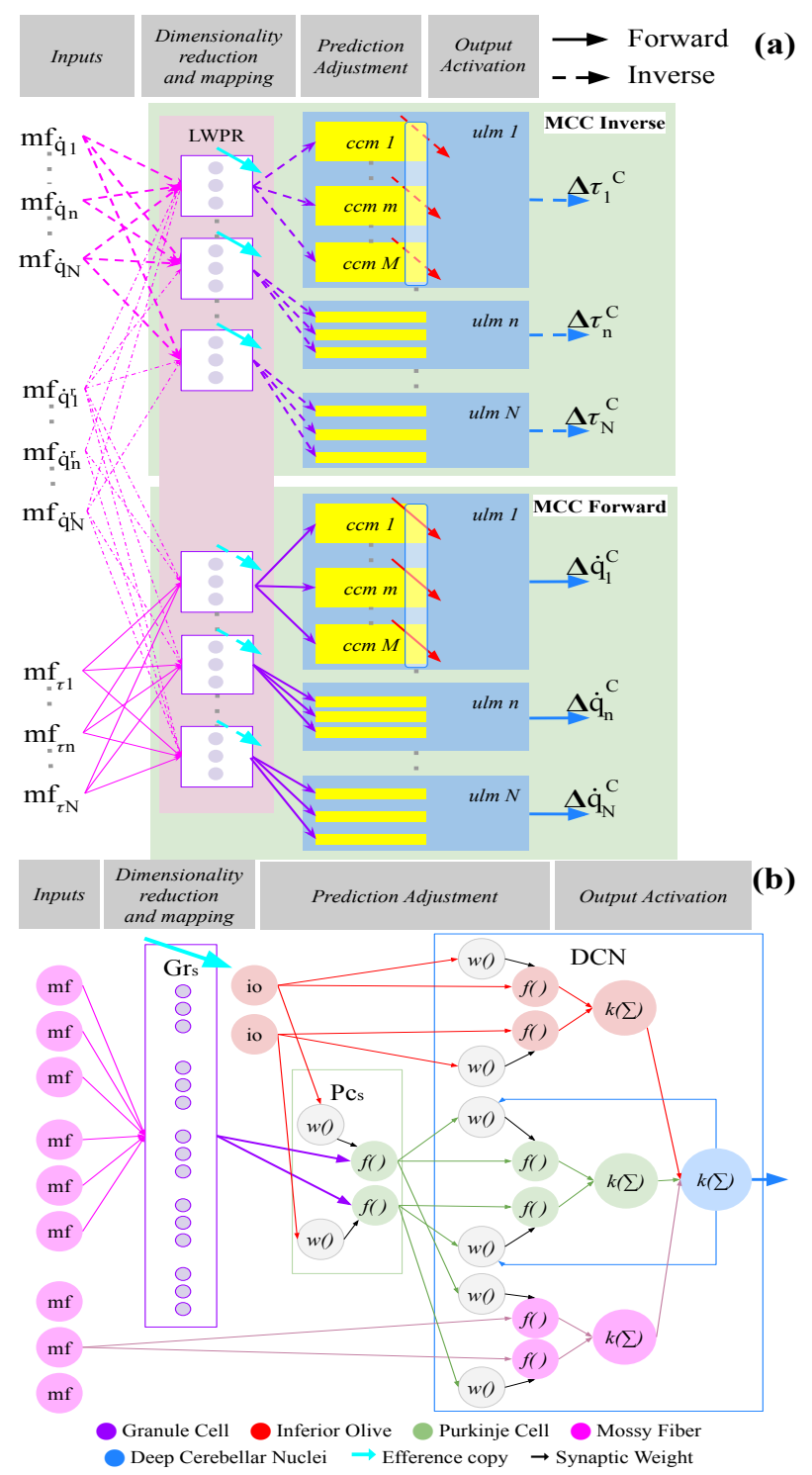

Fig. 4: Cerebellar-like neural network scheme: (a) structural modular partition of the inverse and forward module; (b) details of the networks.

cells, that in Marr's opinion encode combinations of mossy fibers inputs [20]; the pc Purkinje cells (in green Fig.3), that modulated by the inferior olive axon and excited by the pf parallel fibers (in violet) projecting from the granule cells, they influence the activity of the den deep cerebellar nuclei (in blue). The den is inhibited by the pc and excited by both the io and $\mathrm{mf}$, and it is responsible for the final processing of the signal that is sent outside the cerebellar circuit.

In the proposed model (Fig.4.a), each ulm (light blue box) processes the information of the $n$-th controlled object (where $\mathrm{n}=1, \ldots, \mathrm{N})$. Accordingly, the den of the $n$-th ulm outputs the $\Delta \dot{q}_{n}^{c}$ and $\Delta \tau_{n}^{c}$ cerebellar corrections. Each $u l m$ is divided into $\mathrm{M}$ sub-modules representing the ccm canonical cerebellar microcircuit (yellow boxes in Fig.4.a). Each ccm encodes kinematic and/or dynamic features of the $n$-th controlled object, such as angular position and velocity. The $\mathrm{N} u l m$ together compose the MCC Modular Cerebellar Circuit mapping the inverse and forward models of the robotic system (green boxes in Fig.4.a).

Hereafter for the sake of simplicity, the variable $x$ generally recalls the signals $\dot{q}_{n}$ and $\tau_{n}$ propagating inside the two separated networks, and $w$ generally recalls the specific network weight. The mossy fibers of the inverse MCC transmit information about the actual and reference angular velocity of all the controlled joints,

$$
\begin{gathered}
\mathbf{M F}_{2 N \times 1}^{i n v}=\left[m f_{1}^{i n v}, \ldots, m f_{2 N}^{i n v}\right]^{T}= \\
=\left[\dot{q}_{1}^{r}, \ldots, \dot{q}_{N}^{r}, \dot{q}_{1}, \ldots, \dot{q}_{N}\right]^{T},
\end{gathered}
$$

while the mossy fibers of the forward MCC project the signal encoding the reference angular velocities and the latest control inputs (6),

$$
\begin{gathered}
\mathbf{M F}_{2 N \times 1}^{f r w}=\left[m f_{1}^{f r w}, \ldots, m f_{2 N}^{f r w}\right]^{T}= \\
=\left[\dot{q}_{1}^{r}, \ldots, \dot{q}_{N}^{r}, \tau_{1}^{t o t}(t-1), \ldots, \tau_{N}^{t o t}(t-1)\right]^{T} .
\end{gathered}
$$

The mossy fibers signals are then mapped and exploited to predict the $\tau^{t o t}$ control input (inverse MCC) and $\dot{q}$ system state (forward MCC). As proposed by [44], the granule layer is represented by the Locally Weighted Projection Regression algorithm (LWPR) [70]. The LWPR is a fast on-line nonlinear function approximation algorithm suitable for the reduction of high dimensional state space system. To replicate the efference copy theory [39], [71], the LWPR uses a copy of the outgoing $\tau^{\text {tot }}$ (inverse MCC) and actual $\dot{q}$ (forward MCC) as modulatory signals (in cyan Fig.4) to create and train on-line G local linear models, or rather $G r_{g}$ granule cells (where $\mathrm{g}=1, \ldots, \mathrm{G}$ ). These models are employed by the algorithm to make $\hat{\tau}_{n, g}^{g r}, \hat{\dot{q}}_{n, g}^{g r}$ local predictions of the control input (inverse $\mathrm{MCC}$ ) and angular velocity (forward MCC) respectively. The final output of the granular-parallel fibers layer (in violet Fig.4.b) is the weighted mean of all the linear models ( refer to [70] for the complete set of formulas),

$$
\hat{x}_{n}^{p f}=\frac{\sum_{g=1}^{g=G} w_{n, g}^{g r} \cdot \hat{x}_{n, g}^{g r}}{\sum_{g=1}^{g=G} w_{n, g}^{g r}} .
$$

The $w^{p f-p c}$ [42] synaptic strengths of the pf-pc parallel fibers-Purkinje cells connections (Table I) is modulated by the io inferior olive transmitting the error signals $(3,4,5)$ (in red Fig.4.b),

$$
\begin{aligned}
\mathbf{i o}_{n}^{i n v} & =\left[i o_{n, 1}^{i n v}, i o_{n, 2}^{i n v}\right]^{T}=\left[\epsilon_{n}, e_{n}^{t o t}\right]^{T}, \\
\mathbf{i o}_{n}^{f r w} & =\left[i o_{n, 1}^{f r w}, i o_{n, 2}^{f r w}\right]^{T}=\left[\epsilon_{n}, e_{n}^{f b}\right]^{T} .
\end{aligned}
$$

The Purkinje cell output signal (in green Fig.4.b) is the result of the $\hat{x}^{p f}$ modulated LWPR prediction (9),

$$
x_{n, m}^{p c}=w_{n, m}^{p f-p c}\left(t, i o_{n, m}\right) \cdot \hat{x}_{n}^{p f} .
$$

Respect to [44], [52], both the MF mossy fibers input vectors and the $x^{p c}$ Purkinje cells signals are reformulated: the $x^{p f}$ is represented by the final LWPR prediction and not by the linear combination of the network weights; the $x^{p c}$ is the result of a biologically plausible learning rule function of the error $(10,11)$, instead of the direct proportion of the error 
signal; the inferior olive transmits the angular position and velocity error instead of the $\tau^{f b}$ [72].

The pc signal transmitted to the den (in green Fig.4.b) is scaled by the $w_{n, m}^{p c-d c n}$ synaptic weight [42], which is function of both the pc and den activities,

$$
x_{n, m}^{p c-d c n}=w_{n, m}^{p c-d c n}\left(t, x_{n, m}^{p c}, \Delta x_{n}^{c}\right) \cdot x_{n, m}^{p c} .
$$

In the proposed scheme, the mossy fibers (in magenta Fig.4.b) project to the den deep cerebellar nuclei a copy of the $\tau_{n}^{\text {tot }}$ outgoing control input (6) (inverse MCC) and the $\dot{q}_{n}$ actual angular velocity (forward MCC). The mf contribution to the den activity is highly influenced by the pc Purkinje cells [42],

$$
x_{n, m}^{m f-d c n}=w_{n, m}^{m f-d c n}\left(t, x_{n, m}^{p c}\right) \cdot x_{n},
$$

while the strength of the inferior olive input to the deep cerebellar nuclei is determined by the io itself [73],

$$
x_{n, m}^{i o-d c n}=w_{n, m}^{i o-d c n}\left(t, i o_{n, m}\right) \cdot i o_{n, m} .
$$

The final corrective action of each cerebellar-like network (in blue Fig.4.b) is function of the excitatory activity of mossy fibers and inferior olive, and the inhibitory action of the Purkinje cells,

$$
\begin{aligned}
\Delta x_{n}^{c}=+k\left(k\left(\sum_{m=1}^{M} x_{n, m}^{m f-d c n}\right)+\right. & k\left(\sum_{m=1}^{M} x_{n, m}^{i o-d c n}\right)+ \\
& \left.-k\left(\sum_{m=1}^{M} x_{n, m}^{p c-d c n}\right)\right),
\end{aligned}
$$

where the nonlinear activation function $k(y)$ is defined as,

$$
k(y)=\frac{2}{1+e^{-2 y}}-1 .
$$

\section{RESULTS}

Four architectures that differ in terms of internal models contributions are compared: (I) feedback controller; (II) feedback controller combined with inverse cerebellar-like network; (III) feedback controller combined with forward cerebellarlike network; (IV) feedback controller combined with inverse and forward cerebellar-like networks. Each architecture has been tested in the presence of table-ball disturbances (case A), and extra uniformly distributed noise $\mathcal{U}(-0.1,0.1)$ added to the process variables read by the sensors (case B). Hereafter, we use the notation "architecture number $+\mathrm{A} / \mathrm{B}$ " (for instance IIA) to recall a specific test.

The software is based on the ROS messaging framework [74] integrated in the Gazebo-based simulation environment Neurorobotics Platform (NRP) [61]. The three wrist joints are controlled in effort through the Gazebo service ApplyJointEffort. The encoders information are sampled at $f_{\text {samp }}=50 \mathrm{~Hz}$. The computer used for the test has the Ubuntu 16.04 Operating system (OS type $64-$ bit), the Intel Core $^{T M} i 7-7700 H Q$ CPU@ $2.80 \mathrm{GHz} \times 8$ processor, and the GeForce GTX 1050/PCIe/SSE2 graphics card. The tests are performed 20 times per experiment. Due to the stochastic nature of the experiments, the recorded data are expressed as $\mu$ mean value and $\sigma$ standard deviation of the 20 tests. The oscillation $(1,2)$ period is $T=4 \mathrm{sec}$ which corresponds to one $\mathrm{k}$ trial, or rather iteration. The ball is launched from above when the table is parallel to the floor (1st trial). Due to some simulator problems that cause initial jerky movements of the robot, the cerebellar-like networks initiate the learning after the 5 th trial, and are enabled to send correction after 2 more trials. This procedure is generally not necessary and is tailored to the simulator. The paper focuses on the angular position tracking performances of each experiment, which are measured in terms of MAE mean absolute error evolution computed for the $\epsilon_{n}$ angular position error of each joint,

$$
\operatorname{mae}_{q_{n}}(k)=\frac{\sum_{i=t}^{t+T}\left|e_{q_{n}}(i)\right|}{T} \text { for } n=0, \ldots, N .
$$

Fig.5 reports the MAE evolution obtained for the three controlled joint during the 20 tests. From the analysis, the IV architecture (in magenta) resulted to correct larger errors faster with respect to the two networks used independently. This reactive behavior also appears in the III scheme (in cyan),

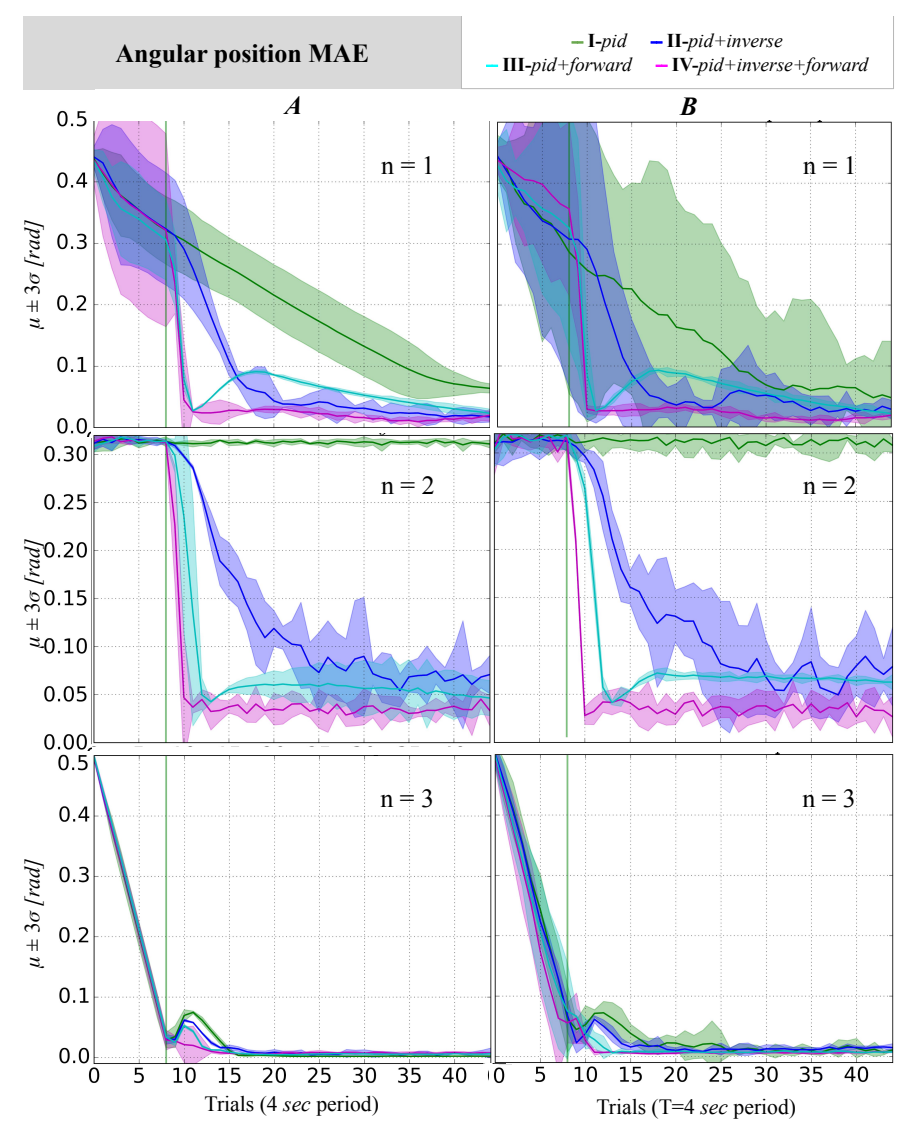

Fig. 5: Control architectures performances. The evolution of the MAE mean absolute error shows how the system is improving its tracking accuracy over the oscillations period. The plot shows the results of the 20 tests in terms of $\mu$ mean value (solid line) and $99.7 \%$ confidence interval (colored area). The vertical green line represent the moment the cerebellum starts adding contributions to the feedback loop ( $k=8$ trial). 
TABLE I: Weighting kernel parameters for $\left[\mathrm{ccm}_{1}, \mathrm{ccm}_{2}\right]$ [42], [59]: LTP long-term potentiation, LTD long-term depression, MTP potentiation modulating term, MTD depression modulating term, $\alpha$ decaying factor.

\begin{tabular}{|c|c|c|c|c|c|c|c|c|c|c|c|c|}
\hline & \multicolumn{6}{|c|}{ PF-PC } & \multicolumn{6}{|c|}{ PC-DCN } \\
\hline & \multicolumn{3}{|c|}{ Forward } & \multicolumn{3}{|c|}{ Inverse } & \multicolumn{3}{|c|}{ Forward } & \multicolumn{3}{|c|}{ Inverse } \\
\hline $\mathrm{n}$ & LTP & LTD & $\alpha$ & LTP & LTD & $\alpha$ & LTP & LTD & $\alpha$ & LTP & LTD & $\alpha$ \\
\hline 1 & {$\left[10^{-2}, 10^{-3.3}\right]$} & {$\left[10^{-2}, 10^{-3.3}\right.$} & {$[800,170]$} & {$\left[10^{-2}, 10^{-3}\right]$} & {$\left[10^{-2}, 10^{-3}\right]$} & {$[300,70]$} & {$\left[10^{-4}, 10^{-4}\right]$} & {$\left[10^{-4}, 10^{-4}\right]$} & {$[2,2]$} & {$\left[10^{-4}, 10^{-4}\right]$} & {$\left[10^{-4}, 10^{-4}\right]$} & {$[2,2]$} \\
\hline 2 & {$\left[10^{-2}, 10^{-3.3}\right]$} & {$\left[10^{-2}, 10^{-3.3}\right.$} & {$[800,170]$} & {$\left[10^{-2}, 10^{-3}\right]$} & {$\left[10^{-2}, 10^{-3}\right]$} & {$[300,70]$} & {$\left[10^{-4}, 10^{-4}\right]$} & {$\left[10^{-4}, 10^{-4}\right]$} & {$[2 ., 2]$} & {$\left[10^{-4}, 10^{-4}\right]$} & {$\left[10^{-4}, 10^{-4}\right]$} & {$[2,2]$} \\
\hline \multirow[t]{3}{*}{3} & {$\left[10^{-2}, 10^{-1}\right]$} & {$\left[10^{-2}, 10^{-1}\right]$} & {$[800,170]$} & {$\left[10^{-2}, 10^{-3}\right]$} & {$\left[10^{-2}, 10^{-3}\right]$} & {$[500,70]$} & {$\left[10^{-4}, 10^{-4}\right]$} & {$\left[10^{-4}, 10^{-4}\right]$} & {$[2 ., 2]$} & {$\left[10^{-4}, 10^{-4}\right]$} & {$\left[10^{-4}, 10^{-4}\right]$} & {$[2 ., 2]$} \\
\hline & \multicolumn{6}{|c|}{ IO-DCN } & \multicolumn{6}{|c|}{ MF-DCN } \\
\hline & \multicolumn{3}{|c|}{ Forward } & \multicolumn{3}{|c|}{ Inverse } & \multicolumn{3}{|c|}{ Forward } & \multicolumn{3}{|c|}{ Inverse } \\
\hline $\mathrm{n}$ & MTP & MTD & $\alpha$ & MTP & MTD & $\alpha$ & LTP & LTD & $\alpha$ & LTP & LTD & $\alpha$ \\
\hline 1 & {$\left[10^{-3}, 10^{-4}\right]$} & {$\left[10^{-4}, 10^{-5}\right]$} & {$[1000,100]$} & {$\left[10^{-3}, 10^{-4}\right]$} & {$\left[10^{-4}, 10^{-5}\right]$} & {$[100,100]$} & {$\left[10^{-4}, 10^{-4}\right]$} & {$\left[10^{-4}, 10^{-4}\right]$} & {$[2,2]$} & {$\left[10^{-4}, 10^{-4}\right]$} & {$\left[10^{-4}, 10^{-4}\right]$} & {$[2,2]$} \\
\hline 2 & {$\left[10^{-3}, 10^{-3}\right]$} & {$\left[10^{-4}, 10^{-4}\right]$} & {$[600,100]$} & {$\left[10^{-3}, 10^{-3}\right]$} & {$\left[10^{-4}, 10^{-4}\right]$} & {$[100,200]$} & {$\left[10^{-4}, 10^{-4}\right]$} & {$\left[10^{-4}, 10^{-4}\right]$} & {$[2 ., 2]$} & {$\left[10^{-4}, 10^{-4}\right]$} & {$\left[10^{-4}, 10^{-4}\right]$} & {$[2,2]$} \\
\hline 3 & {$\left[10^{-4}, 10^{-4}\right]$} & {$\left[10^{-5}, 10^{-5}\right]$} & {$[300,1000]$} & {$\left[10^{-4}, 10^{-4}\right]$} & {$\left[10^{-5}, 10^{-5}\right]$} & {$[500,200]$} & {$\left[10^{-4}, 10^{-4}\right]$} & {$\left[10^{-4}, 10^{-4}\right]$} & {$[2 ., 2]$} & {$\left[10^{-4}, 10^{-4}\right]$} & {$\left[10^{-4}, 10^{-4}\right]$} & {$[2,2]$} \\
\hline
\end{tabular}

but it is highly boosted in IV by the feed forward correction of the inverse model, thanks to the reduction of the loop delays affecting the feedback controller. Although the feedback controller is highly perturbed by the propagation of noise in the system (IB in green Fig. 5), the biomimetic architectures do not present evident consequences. In particular, thanks to the forward model action, architectures III and IV robustly reduce the effect of noise as suggested by [51].

In about 5 trials, architecture IV rapidly corrects the feedback controller performance leading to an extra drop of $12 \%$ (joint 1) and $90 \%$ (joint 2) with respect to architecture I (in green). Moreover, architecture IV is the only one able to correct rapidly the deviation of joint 3 between trials 8 and 15. It is worthwhile to mention that the feedback controller of the first joint is highly affected by the table weight, which slowly leads the joint towards the correct reference.

As we expected, the number of Gr granule cells of each ulm (LWPR receptive fields) created during the test case $\mathbf{B}$ are larger respect to the not noisy test (Table II), especially for architecture III and IV that resulted to act more robustly against noise. Moreover, We believe that the large numerical difference between the Gr of the two MCC is due to the update of the forward MCC before the inverse MCC that solve the one-to-many mapping issue of the inverse model [55].

\section{CONCLUSIONS}

Thus far, we have presented, tested, and compared four control architectures based on a versatile and real-time modeling structure that replicates the cerebellar internal models individual and combinatorial theories. In particular, we introduced a novel bio-mimetic learning and control cerebellar network that combines computational neuroscience, machine learning, and artificial neural network methods. The biomimetic controllers are tested on a humanoid robot acting in a perturbed nondeterministic environment.

The experiments confirmed the theories about the internal model independent and combinatorial contribution. In particular, the forward model resulted to act rapidly and efficiently against any noise and external perturbation in the early adaptation period, while the inverse model highly

TABLE II: Receptive fields created by the LWPR.

\begin{tabular}{|c|c|c|c|c|c|c|}
\hline \multirow{2}{*}{$\begin{array}{l}\text { Architecture } \\
\text { Case }\end{array}$} & \multicolumn{2}{|c|}{ II } & \multicolumn{2}{|c|}{ III } & \multicolumn{2}{|c|}{ IV } \\
\hline & $\mathrm{A}$ & $\mathrm{B}$ & A & B & $\mathrm{A}$ & $\mathrm{B}$ \\
\hline Inverse MCC & 8 & 14 & - & - & 11 & 16 \\
\hline Forward MCC & - & 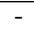 & 78 & 146 & 436 & 510 \\
\hline
\end{tabular}

boosted the feedback controller action through the adaptive feed forward compensation that helped to reduce tracking error and loop delays in the post adaptation period. Finally, the combination of the internal models into a feedback control scheme merged the models strengths and guaranteed improved tracking accuracy, adaptation, high robustness to perturbations and filtering of noise.

In the details of the biomimetic neural network, the granule cells-parallel fibers layers are artificially interpreted with the LWPR. The algorithm has been employed differently with respect to the past experiments [75], [44], both in terms of input, training, and output signals (the details are listed in Section II). The algorithm resulted an efficient tool for the online mapping of high dimensional input space, and prediction of complex non-linear functions. Although high estimation errors can lead the LWPR to jerky prediction, the stratified structure of the network and the application of nonlinear functions largely damped any over-reactive behaviors.

Most of the artificial cerebellar model do not employ biologically plausible learning and frequently miss the dcn deep cerebellar nuclei layer [76], [51], [44]. In the proposed model, the learning rules that iteratively update the network weights are based on synaptic plasticities derived from computational neuroscience studies [42], [59]. At some extend, this learning approach makes the network conceptually closer to the spiking models presented in the literature [42], [57], [77] but still not comparable due to the different codification of the signals propagating inside the networks. Moreover with respect to [57], we embedded the networks into a feedback control scheme where the MCCs are combined in a "tandem model" [55], in order to solve the one-to-many mapping problem affecting the inverse model. Although the high number of synaptic plasticities includes a large number of learning parameters, the network does not required excessive tuning, and no adjustment were necessary between experiments: the tuning resulted to work robustly even in highly noisy conditions (experiments B). For the scope of this manuscript, we did not focus on the optimal tuning, but we believe, as in most neural network case, that a modeling algorithm could help assetting the best combination of parameters. An additional difference with respect to the literature, is the internal modularity of each uml unit learning machine, which is divided in specialized compartment encoding kinematics or dynamical features. This modular and layered structure of the network significantly reduced the effects of nonlinearities, sensor noise, high feedback and prediction (LWPR) errors. 
Despite the novelties with respect to past models, at the current state the cerebellar network can not generalize all the possible conditions. The model itself replicate only a small component of a wider and modular structure that is under investigation. The biological cerebellum is composed by a large number of canonical circuits working in parallel. It is not clear how different circuits operate together towards a more general and complete learning and control action, but it is our intention to analyze these motor dynamics employing the proposed component as main building block. Moreover, the cerebellum is just one of the central nervous system area involved into the neural control of movements. We believe that the full exploitation of the cerebellar potentialities is only possible if the circuit collaborates with other generalized and biological plausible control structures.

\section{ACKNOWLEDGMENT}

This work has received funding from the EU-H2020 Framework Program for Research and Innovation under the specific grant agreement No. 785907 (Human Brain Project SGA2), and from the Marie Curie project n. 705100 (Biomodular).

\section{REFERENCES}

[1] J. Nakanishi, R. Cory, M. Mistry, J. Peters, and S. Schaal, "Operational space control: A theoretical and empirical comparison," The International Journal of Robotics Research, vol. 27, no. 6, pp. 737-757, 2008.

[2] C. G. Atkeson, C. H. An, and J. M. Hollerbach, "Estimation of inertial parameters of manipulator loads and links," The International Journal of Robotics Research, vol. 5, no. 3, pp. 101-119, 1986.

[3] J.-A. Ting, M. Mistry, J. Peters, S. Schaal, and J. Nakanishi, "A bayesian approach to nonlinear parameter identification for rigid body dynamics.", in Robotics: Science and Systems, pp. 32-39, Philadelphia, USA, 2006.

[4] K. S. Narendra and A. M. Annaswamy, "Persistent excitation in adaptive systems," International Journal of Control, vol. 45, no. 1, pp. 127-160, 1987.

[5] R. M. Sanner and J.-J. Slotine, "Gaussian networks for direct adaptive control," IEEE Transactions on neural networks, vol. 3, no. 6, pp. 837863, 1992.

[6] D. Nguyen-Tuong and J. Peters, "Model learning for robot control: a survey," Cognitive processing, vol. 12, no. 4, pp. 319-340, 2011.

[7] J. A. Farrell and M. M. Polycarpou, Adaptive approximation based control: unifying neural, fuzzy and traditional adaptive approximation approaches, vol. 48. John Wiley \& Sons, 2006.

[8] H. D. Patino, R. Carelli, and B. R. Kuchen, "Neural networks for advanced control of robot manipulators," IEEE Transactions on Neural networks, vol. 13, no. 2, pp. 343-354, 2002.

[9] J. Kocijan, R. Murray-Smith, C. E. Rasmussen, and A. Girard, "Gaussian process model based predictive control," in Proceedings of the 2004 American Control Conference, vol. 3, pp. 2214-2219, IEEE, 2004.

[10] J. Nakanishi and S. Schaal, "Feedback error learning and nonlinear adaptive control," Neural Networks, vol. 17, no. 10, pp. 1453-1465, 2004.

[11] K. S. Narendra and S. Mukhopadhyay, "Associative learning in random environments using neural networks," IEEE transactions on neural networks, vol. 2, no. 1, pp. 20-31, 1991.

[12] F. H. Glanz, W. T. Miller, and L. G. Kraft, "An overview of the cmac neural network," in [1991 Proceedings] IEEE Conference on Neural Networks for Ocean Engineering, pp. 301-308, IEEE, 1991.

[13] T. Zhang, S. S. Ge, and C. C. Hang, "Adaptive neural network control for strict-feedback nonlinear systems using backstepping design," Automatica, vol. 36, no. 12, pp. 1835-1846, 2000

[14] W. He, B. Huang, Y. Dong, Z. Li, and C.-Y. Su, "Adaptive neural network control for robotic manipulators with unknown deadzone," IEEE transactions on cybernetics, vol. 48, no. 9, pp. 2670-2682, 2018.

[15] J. Weng, C. Evans, W. S. Hwang, and Y.-B. Lee, "The developmental approach to artificial intelligence: Concepts, developmental algorithms and experimental results," in NSF Design and Manufacturing Grantees Conference, Long Beach, CA, 1999.
[16] D. Vernon, G. Metta, and G. Sandini, "A survey of artificial cognitive systems: Implications for the autonomous development of mental capabilities in computational agents," IEEE transactions on evolutionary computation, vol. 11, no. 2, pp. 151-180, 2007.

[17] G. Metta, G. Sandini, and J. Konczak, "A developmental approach to visually-guided reaching in artificial systems," Neural networks, vol. 12, no. 10, pp. 1413-1427, 1999.

[18] D. Luo, F. Hu, T. Zhang, Y. Deng, and X. Wu, "How does a robot develop its reaching ability like human infants do?," IEEE Transactions on Cognitive and Developmental Systems, vol. 10, no. 3, pp. 795-809, 2018.

[19] J. S. Albus, "A new approach to manipulator control: The cerebellar model articulation controller (cmac)," Journal of Dynamic Systems, Measurement, and Control, vol. 97, no. 3, pp. 220-227, 1975.

[20] D. Marr, "A theory of cerebellar cortex," The Journal of physiology, vol. 202 , no. 2, pp. 437-470, 1969.

[21] J. S. Albus, "A theory of cerebellar function," Mathematical Biosciences, vol. 10, no. 1-2, pp. 25-61, 1971.

[22] P. L. Strick, R. P. Dum, and J. A. Fiez, "Cerebellum and nonmotor function," Annual review of neuroscience, vol. 32, pp. 413-434, 2009.

[23] L. F. Koziol, D. E. Budding, and D. Chidekel, "From movement to thought: executive function, embodied cognition, and the cerebellum," The Cerebellum, vol. 11, no. 2, pp. 505-525, 2012.

[24] D. Caligiore, G. Pezzulo, R. C. Miall, and G. Baldassarre, "The contribution of brain sub-cortical loops in the expression and acquisition of action understanding abilities," Neuroscience \& Biobehavioral Reviews, vol. 37, no. 10, pp. 2504-2515, 2013.

[25] M. Manto, J. M. Bower, A. B. Conforto, J. M. Delgado-García, S. N. F. Da Guarda, M. Gerwig, C. Habas, N. Hagura, R. B. Ivry, P. Mariën, et al., "Consensus paper: roles of the cerebellum in motor controlthe diversity of ideas on cerebellar involvement in movement," The Cerebellum, vol. 11, no. 2, pp. 457-487, 2012.

[26] J. C. Houk and S. P. Wise, "Distributed modular architectures linking basal ganglia, cerebellum, and cerebral cortex: their role in planning and controlling action," Cerebral cortex, vol. 5, no. 2, pp. 95-110, 1995.

[27] D. Caligiore, G. Pezzulo, G. Baldassarre, A. C. Bostan, P. L. Strick, K. Doya, R. C. Helmich, M. Dirkx, J. Houk, H. Jörntell, et al., "Consensus paper: towards a systems-level view of cerebellar function: the interplay between cerebellum, basal ganglia, and cortex," The Cerebellum, vol. 16, no. 1, pp. 203-229, 2017.

[28] R. Miall, D. J. Weir, D. M. Wolpert, and J. Stein, "Is the cerebellum a smith predictor?," Journal of motor behavior, vol. 25, no. 3, pp. 203216, 1993.

[29] M. Kawato, "Internal models for motor control and trajectory planning," Current Opinion in Neurobiology, vol. 9, no. 6, pp. 718 - 727, 1999.

[30] D. M. Wolpert, R. C. Miall, and M. Kawato, "Internal models in the cerebellum," Trends in cognitive sciences, vol. 2, no. 9, pp. 338-347, 1998.

[31] L. S. Popa, A. L. Hewitt, and T. J. Ebner, "Purkinje cell simple spike discharge encodes error signals consistent with a forward internal model," The Cerebellum, vol. 12, no. 3, pp. 331-333, 2013.

[32] M. Kawato, K. Furukawa, and R. Suzuki, "A hierarchical neural-network model for control and learning of voluntary movement," Biological cybernetics, vol. 57, no. 3, pp. 169-185, 1987.

[33] M. I. Jordan and D. E. Rumelhart, "Forward models: Supervised learning with a distal teacher," Cognitive science, vol. 16, no. 3, pp. 307-354, 1992.

[34] N. Schweighofer, M. A. Arbib, and M. Kawato, "Role of the cerebellum in reaching movements in humans. i. distributed inverse dynamics control," European Journal of Neuroscience, vol. 10, no. 1, pp. 86-94, 1998.

[35] J. F. Medina, "The multiple roles of purkinje cells in sensori-motor calibration: to predict, teach and command," Current opinion in neurobiology, vol. 21, no. 4, pp. 616-622, 2011.

[36] B. Köster, G. Deuschl, M. Lauk, J. Timmer, B. Guschlbauer, and C. Lücking, "Essential tremor and cerebellar dysfunction: abnormal ballistic movements," Journal of Neurology, Neurosurgery \& Psychiatry, vol. 73, no. 4, pp. 400-405, 2002.

[37] A. G. Feldman, "New insights into action-perception coupling," Experimental brain research, vol. 194, no. 1, pp. 39-58, 2009.

[38] K. Friston, "What is optimal about motor control??" Neuron, vol. 72, no. 3, pp. 488-498, 2011.

[39] E. Todorov and M. I. Jordan, "Optimal feedback control as a theory of motor coordination," Nature neuroscience, vol. 5, no. 11, p. 1226, 2002.

[40] M. Kawato, "Feedback-error-learning neural network for supervised motor learning," in Advanced neural computers, pp. 365-372, Elsevier, 1990. 
[41] A. G. Barto, A. H. Fagg, N. Sitkoff, and J. C. Houk, "A cerebellar model of timing and prediction in the control of reaching," Neural computation, vol. 11, no. 3, pp. 565-594, 1999.

[42] J. A. Garrido Alcazar, N. R. Luque, E. DAngelo, and E. Ros, "Distributed cerebellar plasticity implements adaptable gain control in a manipulation task: a closed-loop robotic simulation," Frontiers in neural circuits, vol. 7, p. 159, 2013.

[43] N. R. Luque, J. A. Garrido, F. Naveros, R. R. Carrillo, E. D’Angelo, and E. Ros, "Distributed cerebellar motor learning: A spike-timingdependent plasticity model," Frontiers in Computational Neuroscience, vol. 10, p. 17, 2016.

[44] S. Tolu, M. Vanegas, N. R. Luque, J. A. Garrido, and E. Ros, "Bioinspired adaptive feedback error learning architecture for motor control," Biological Cybernetics, vol. 106, no. 8-9, pp. 507-522, 2012.

[45] L. Vannucci, E. Falotico, S. Tolu, V. Cacucciolo, P. Dario, H. H. Lund, and C. Laschi, "A comprehensive gaze stabilization controller based on cerebellar internal models," Bioinspiration \& biomimetics, vol. 12, no. 6 , p. 065001, 2017.

[46] R. C. Miall and D. M. Wolpert, "Forward models for physiological motor control," Neural networks, vol. 9, no. 8, pp. 1265-1279, 1996.

[47] S. Kitazawa, T. Kimura, and P.-B. Yin, "Cerebellar complex spikes encode both destinations and errors in arm movements," Nature, vol. 392, no. 6675, p. 494, 1998.

[48] H. Imamizu, S. Miyauchi, T. Tamada, Y. Sasaki, R. Takino, B. PuÈtz, T. Yoshioka, and M. Kawato, "Human cerebellar activity reflecting an acquired internal model of a new tool," Nature, vol. 403, no. 6766, p. 192, 2000.

[49] D. J. Serrien and M. Wiesendanger, "Grip-load force coordination in cerebellar patients," Experimental brain research, vol. 128, no. 1-2, pp. 76-80, 1999.

[50] D. A. Nowak, H. Topka, D. Timmann, H. Boecker, and J. Hermsdörfer, "The role of the cerebellum for predictive control of grasping," The Cerebellum, vol. 6, no. 1, p. 7, 2007.

[51] J. Porrill and P. Dean, "Recurrent cerebellar loops simplify adaptive control of redundant and nonlinear motor systems," Neural computation, vol. 19, no. 1, pp. 170-193, 2007.

[52] S. Tolu, M. Vanegas, J. A. Garrido, N. R. Luque, and E. Ros, "Adaptive and predictive control of a simulated robot arm," Int. J. Neural Syst., vol. 23, no. 3, 2013.

[53] D. M. Wolpert and M. Kawato, "Multiple paired forward and inverse models for motor control," Neural networks, vol. 11, no. 7-8, pp. 13171329, 1998.

[54] M. Mischiati, H.-T. Lin, P. Herold, E. Imler, R. Olberg, and A. Leonardo, "Internal models direct dragonfly interception steering," Nature, vol. 517, no. 7534 , p. $333,2015$.

[55] T. Honda, S. Nagao, Y. Hashimoto, K. Ishikawa, T. Yokota, H. Mizusawa, and M. Ito, "Tandem internal models execute motor learning in the cerebellum," Proceedings of the National Academy of Sciences, vol. 115, no. 28, pp. 7428-7433, 2018.

[56] E. Escobar-Juárez, G. Schillaci, J. Hermosillo-Valadez, and B. LaraGuzmán, "a self-organized internal models architecture for coding sensory-motor schemes," Frontiers in Robotics and AI, vol. 3, p. 22, 2016.

[57] N. R. Luque, J. A. Garrido, R. R. Carrillo, S. Tolu, and E. Ros, "Adaptive cerebellar spiking model embedded in the control loop: context switching and robustness against noise," International Journal of Neural Systems, vol. 21, no. 05, pp. 385-401, 2011.

[58] T. DeWolf, T. C. Stewart, J.-J. Slotine, and C. Eliasmith, "A spiking neural model of adaptive arm control," Proceedings of the Royal Society B: Biological Sciences, vol. 283, no. 1843, p. 20162134, 2016.

[59] N. R. Luque, J. A. Garrido, R. R. Carrillo, E. D'Angelo, and E. Ros, "Fast convergence of learning requires plasticity between inferior olive and deep cerebellar nuclei in a manipulation task: a closed-loop robotic simulation," Frontiers in computational neuroscience, vol. 8, p. 97, 2014.

[60] G. Metta, G. Sandini, D. Vernon, L. Natale, and F. Nori, "The icub humanoid robot: An open platform for research in embodied cognition," in Proceedings of the 8th Workshop on Performance Metrics for Intelligent Systems, PerMIS '08, (New York, NY, USA), pp. 50-56, ACM, 2008.

[61] E. Falotico, L. Vannucci, A. Ambrosano, U. Albanese, S. Ulbrich, J. C. Vasquez Tieck, G. Hinkel, J. Kaiser, I. Peric, O. Denninger, et al., "Connecting artificial brains to robots in a comprehensive simulation framework: The neurorobotics platform," Frontiers in neurorobotics, vol. 11, p. 2, 2017.

[62] G. Hinkel, H. Groenda, S. Krach, L. Vannucci, O. Denninger, N. Cauli, S. Ulbrich, A. Roennau, E. Falotico, M.-O. Gewaltig, et al., "A framework for coupled simulations of robots and spiking neuronal networks,"
Journal of Intelligent \& Robotic Systems, vol. 85, no. 1, pp. 71-91, 2017.

[63] M. Ito, The cerebellum and neural control. Raven press, 1984

[64] H. Miyamoto, M. Kawato, T. Setoyama, and R. Suzuki, "Feedback-errorlearning neural network for trajectory control of a robotic manipulator," Neural networks, vol. 1, no. 3, pp. 251-265, 1988.

[65] M. J. Wagner and M. A. Smith, "Shared internal models for feedforward and feedback control," Journal of Neuroscience, vol. 28, no. 42, pp. 10663-10673, 2008.

[66] J. Munuera, P. Morel, J.-R. Duhamel, and S. Deneve, "Optimal sensorimotor control in eye movement sequences," Journal of Neuroscience, vol. 29, no. 10, pp. 3026-3035, 2009.

[67] E. DAngelo, A. Antonietti, S. Casali, C. Casellato, J. A. Garrido, N. R. Luque, L. Mapelli, S. Masoli, A. Pedrocchi, F. Prestori, M. F. Rizza, and E. Ros, "Modeling the cerebellar microcircuit: New strategies for a long-standing issue," Frontiers in Cellular Neuroscience, vol. 10, p. 176, 2016.

[68] T. J. Ebner, A. L. Hewitt, and L. S. Popa, "What features of limb movements are encoded in the discharge of cerebellar neurons?," The Cerebellum, vol. 10, pp. 683-693, Dec 2011.

[69] C. I. De Zeeuw, C. C. Hoogenraad, S. Koekkoek, T. J. Ruigrok, N. Galjart, and J. I. Simpson, "Microcircuitry and function of the inferior olive," Trends in neurosciences, vol. 21, no. 9, pp. 391-400, 1998

[70] S. Vijayakumar and S. Schaal, "Locally weighted projection regression: An o (n) algorithm for incremental real time learning in high dimensional space," in Proceedings of the Seventeenth International Conference on Machine Learning (ICML 2000), vol. 1, pp. 288-293, 2000.

[71] S. H. Scott, "The computational and neural basis of voluntary motor control and planning," Trends in Cognitive Sciences, vol. 16, no. 11, pp. $541-549,2012$.

[72] K. Doya, H. Kimura, and M. Kawato, "Neural mechanisms of learning and control," IEEE Control Systems Magazine, vol. 21, no. 4, pp. 42-54, 2001.

[73] N. R. Luque, R. R. Carrillo, F. Naveros, J. A. Garrido, and M. Sez-Lara, "Integrated neural and robotic simulations. simulation of cerebellar neurobiological substrate for an object-oriented dynamic model abstraction process," Robotics and Autonomous Systems, vol. 62, no. 12, pp. 1702 - 1716, 2014.

[74] M. Quigley, K. Conley, B. Gerkey, J. Faust, T. Foote, J. Leibs, R. Wheeler, and A. Y. Ng, "Ros: an open-source robot operating system," in ICRA workshop on open source software, vol. 3, p. 5, Kobe, Japan, 2009.

[75] L. Lonini, L. Dipietro, L. Zollo, E. Guglielmelli, and H. I. Krebs, "An internal model for acquisition and retention of motor learning during arm reaching," Neural computation, vol. 21, no. 7, pp. 2009-2027, 2009.

[76] W. Miller, "Sensor-based control of robotic manipulators using a general learning algorithm," IEEE Journal on Robotics and Automation, vol. 3, no. 2, pp. 157-165, 1987.

[77] C. Casellato, A. Antonietti, J. A. Garrido, R. R. Carrillo, N. R. Luque, E. Ros, A. Pedrocchi, and E. D'Angelo, "Adaptive robotic control driven by a versatile spiking cerebellar network," PLOS ONE, vol. 9, pp. 1-17, 112014. 